\title{
Führt Sprachkompetenz zu zielorientierteren und erfolgreicheren Studierenden? Eine Motivationsstudie bei Studierenden
}

Julienne Schult, BSc

Institut für Zoologie

Universität Innsbruck

ao. Univ.-Prof. Dr. Thorsten Schwerte

Institut für Zoologie

Universität Innsbruck

\section{Einleitung}

\subsection{Die Berufswahl}

Die Berufswahl stellt eine wichtige Entscheidung dar. In der Forschung gibt es diverse Ansätze zu dem Prozess der Berufsfindung und zu den Faktoren, die diese Entscheidung beeinflussen. Es sind viele verschiedene Variablen, die die Berufswahl beeinflussen, wie zum Beispiel die soziale Herkunft, geographische Region, Schule und Ausbildung (Kleinbeck 1975). In der Adoleszenz ist die Auseinandersetzung mit und die Entscheidung über die berufliche Laufbahn eine wichtige Entwicklungsaufgabe (Hellberg 2009). Dabei stellt die Studienwahl für viele SchülerInnen mit dem Abschluss der Hochschulreife eine der wichtigen Entscheidungen in ihrer beruflichen Laufbahn dar. Häufig treffen diese jedoch die Wahl nicht ausreichend konsequent, wie die Zahlen der StudienabbrecherInnen zeigen. Im Jahr 2011 betrug die Abbruchquote bei Studierenden in Österreich 35\% und in Deutschland ca. 25\% (OECD 2013). Ein Studium abzubrechen, ist ein komplexer Entscheidungsprozess, der ebenfalls von verschiedenen 
Faktoren beeinflusst wird (Unger et al. 2009). Die Gründe für einen Studienabbruch sind sehr vielfältig und können sehr individuell sein. Wie verschiedene Studien zeigen, gibt es aber einige Gründe, die auf ein Kollektiv von Studierenden zutreffen. Unter anderem gehören Erwerbstätigkeit, fehlendes Engagement, Distanz zur Universität oder Theorielastigkeit des Studiums zu Abbruchgründen (Kolland 2002). Die Frage, warum Studierende ihr Studium abbrechen, ist eine wichtige für die gesamte Gesellschaft, da es die Studierenden selbst wie auch das universitäre Ausbildungssystem belastet, indem u.a. Ressourcen und Platz beansprucht werden. Mögliche Antworten auf diese Frage könnten dabei helfen, Gegenmaßnahmen zu finden, um die Abbruchquoten zu reduzieren (Unger et al. 2009). Maßnahmen gegen einen Abbruch können z.B. bereits vor dem Beginn eines Studiums oder in der Studieneingangsphase ansetzen. Hier wären zum Beispiel der Kontakt zwischen Universitäten und Schulen oder TutorInnen- und MentorInnensysteme zu nennen (Ziegele 1997). Um solche Strategien umzusetzen und zu optimieren, ist es wichtig, die Motivation von SchülerInnen für die Aufnahme eines bestimmten Studiums zu kennen. Diese Informationen können helfen, einen Studiengang attraktiver zu gestalten oder über diesen vorher besser zu informieren und damit höhere Abschlussquoten zu erreichen.

Neben der Frage nach den Gründen eines Studienabbruchs sollte die Frage, was eine/n Studierende/n befähigt, zielorientiert und erfolgreich im Studium zu sein, betrachtet werden, da anzunehmen ist, dass diese Punkte den Studienerfolg erhöhen können. Ein Tool zur Studienerfolgsprognose bei Erstsemestrigen ist sehr erstrebenswert. Dies zeigen unter anderem Untersuchungen verschiedener Wissenschaftler. Freyer et al. (2014) testeten zum Beispiel die Prognose zum Studienerfolg im Fach Chemie an der Universität Duisburg-Essen. Dabei untersuchten sie den Studienerfolg anhand einer Auswertung der Punktezahl in der Klausur zur allgemeinen Chemie mit Hilfe einer linearen Regressionsanalyse. Die Daten wurden anhand von zwei Tests und der Klausurnote erhoben. Am Beginn des Semesters wurde ein Pre-Test durchgeführt. Dieser umfasste einen Test zum schlussfolgernden Denken, einen Fragebogen zum Fachinteresse sowie einige Fragen zu demografischen Angaben, zur Abiturgesamtnote und die Frage nach dem Wunschstudienfach. Ein Post-Test erfolgte am Ende des Semesters, in dem das Fachwissen und Fachinteresse erneut erhoben wurden. Bei der Unter-

Barbara Hinger (Hg.), Zweite „Tagung der Fachdidaktik“ 2015.

Sprachsensibler Sach-Fach-Unterricht - Sprachen im Sprachunterricht.

(C) 2016 innsbruck university press, ISBN 978-3-903122-51-2, DOI 10.15203/3122-51-2 
suchung stellte sich heraus, dass die Prädiktoren Vorwissen, Abiturgesamtnote, Fähigkeit im schlussfolgernden Denken, Fachinteresse und Studienbeitrag einen signifikanten Beitrag zur Prognose des Studienerfolgs liefern. Lediglich die motivationale Variable Wunschfach trug nicht signifikant zur Erklärung des Studienerfolgs bei. Allerdings interagiert die Variable Wunschfach mit den anderen Prädiktoren wie dem Vorwissen, schlussfolgerndes Denken oder der Abiturgesamtnote und kann als Verknüpfungspunkt zwischen diesen angesehen werden. Dies weist darauf hin, dass die Motivation den Einfluss von Vorwissen und kognitiven Fähigkeiten auf den Studienerfolg steuert (Freyer et al. 2014). Ein weiteres Beispiel ist das US-amerikanische Modell zum Studienerfolg, das Kuh et al. (2007) entwickelten. Das Modell zeigt auf, dass der Weg zum Studienerfolg nicht geradlinig verläuft, sondern dass er von Kurven, Umwegen und Sackgassen gekennzeichnet ist. Die Motivation scheint im Feld „Verhalten von StudentInnen" auf. Sie nimmt damit eine zentrale Stellung ein und wird von verschiedenen Aspekten beeinflusst, wie zum Beispiel von schulischem und familiärem Einfluss oder der College-Bereitschaft der Studierenden (Kuh et al. 2007).

An diesen beiden Beispielen wird deutlich, dass sich eine Studienerfolgsprognose einfach quantifizierbarer Parameter, wie der Abiturnote, bedienen kann oder eher qualitativer Parameter, wie der Motivation oder sozialer Aspekte. Motivation ist nicht leicht zu fassen, da Motivation multifaktoriell ist, weshalb auch keine homogenen Erklärungen zur Verfügung stehen. Vielmehr ist Motivation abstrakt zu fassen und unterschiedlichste Prozesse im Hintergrund sind miteinzubeziehen (Rheinberg 2012). In diesem Sinne sind die Gründe, die zu einem Studienabbruch führen, ebenfalls multifaktoriell.

In der hier vorgelegten Untersuchung, die als Pilotstudie angelegt ist, werden die Motive untersucht, die Studierende dazu veranlassen, ein Studium der Biologie an der Universität Innsbruck aufzunehmen. Als Motivation werden hier Faktoren angesehen, die die Entscheidung der Studierenden für ein Biologiestudium beeinflussen, wie z.B. die Vorbildfunktion der Eltern oder die Schule mit anregendem und interessanten Unterricht, aber auch der/die Studierende selbstdurch seine Persönlichkeitsfaktoren wie Begabung und individuelle Interessen. Zur Erhebung der unterschiedlichen Faktoren dienen Motivationsschreiben der Erstsemestrigen des Bachelorstudiums Biologie. Dieses erlaubt sowohl eine

Barbara Hinger (Hg.), Zweite „Tagung der Fachdidaktik“ 2015.

Sprachsensibler Sach-Fach-Unterricht - Sprachen im Sprachunterricht.

(C) 2016 innsbruck university press, ISBN 978-3-903122-51-2, DOI 10.15203/3122-51-2 
Analyse der (fachbezogenen) Sprachkompetenz als auch der Motivation. Es soll weiterhin betrachtet werden, welche Information aus einem Motivationsschreiben für Studienerfolgsprognosen und für weitere Analysen geeignet sein könnte. Eine mögliche frühe Erkennbarkeit von Tendenzen zu einem der fünf BiologieMasterstudiengänge der Universität Innsbruck war ein fakultätspolitischer Parameter. Insbesondere steht dabei das Studium der Zoologie im Blickpunkt. Im vorliegenden Beitrag soll jedoch betrachtet werden, ob die Sprachkompetenz der Studierenden als multifaktorielle Größe sich als möglicher Prädiktor für den Studienerfolg eignet.

\section{Methoden}

\subsection{Das Motivationsschreiben}

Als Aufgabe während der Vorlesung „743101 Skills I“ im Wintersemester 2013/14 sollten die Erstsemestrigen des Bachelorbiologiestudiums ein Motivationsschreiben verfassen, in dem sie ihre Entscheidung für ein Biologiestudium an der Universität Innsbruck schriftlich reflektieren. Die Teilnahme war freigestellt. Die Studierenden konnten dabei Zusatzpunkte für die Gesamtnote erzielen. Das Schreiben sollte maximal zwei DIN A4 Seiten umfassen. Eine Power-Point-Präsentation während der Vorlesung mit Hinweisen zur Verfassung von Motivationsschreiben diente als Hilfestellung. Unter anderem waren in der Präsentation Themen gelistet, die in einem Motivationsschreiben relevant sind, wie die Motivation für das Studium oder die Berufsperspektiven. Diese Motivationsschreiben sind die Datengrundlage der hier vorgestellten Pilot-Studie. Insgesamt basiert die Studie auf 276 Motivationsschreiben, davon wurden 180 Schreiben von Studentinnen und 96 Schreiben von Studenten verfasst. Die Motivationsschreiben wurden von einer Person anhand eines Fragebogens kodiert und ausgewertet, wie in Kapitel 2.2. genauer erläutert wird.

Barbara Hinger (Hg.), Zweite „Tagung der Fachdidaktik“ 2015.

Sprachsensibler Sach-Fach-Unterricht - Sprachen im Sprachunterricht.

(C) 2016 innsbruck university press, ISBN 978-3-903122-51-2, DOI 10.15203/3122-51-2 


\subsection{Der Fragebogen}

Der Fragebogen zur Auswertung der Motivationsschreiben besteht aus zwei Teilen (s. Anhang Tab. 1 und 2). Der erste Teil umfasst die Textbeurteilung und behandelt vier Unterpunkte, die sich am Textqualitäten-Modell nach Nussbaumer \& Sieber (1994) orientieren. Der erste Unterpunkt betrifft die Grundgrößen und orientiert sich daran, ob im Schreiben ein roter Faden vorhanden ist. Danach wird die Sprachrichtigkeit betrachtet. Als dritter Punkt wird die sprachliche Angemessenheit analysiert, zum Beispiel die der Aufgabenstellung angemessene Wortwahl und der Satzbau. Als letzter Punkt wird die inhaltliche Relevanz beurteilt, das heißt die Überzeugungskraft des Motivationsschreibens (Becker-Mrotzek \& Böttcher 2006). Durch die Textbeurteilung konnten die Studierenden maximal 12 Punkte erzielen. Bei den einzelnen Aspekten erhielten sie jeweils einen Punkt, wenn alles als korrekt eingestuft wurde; lag eine mittelmäßige Korrektheit vor, wurde ein halber Punkt vergeben; wenn keine Korrektheit gegeben war, wurde kein Punkt zugeteilt. Für weitere Analysen wurde ein Notenschlüssel verwendet. Erreichte der Studierende ein bis fünf Punkte, wurde das Motivationsschreiben als schlecht eingestuft, lagen sechs bis neun Punkte vor, handelte es sich um ein mittelmäßiges und bei zehn bis zwölf um ein sehr gutes Motivationsschreiben.

Der zweite Teil des Fragebogens bezieht sich auf die Auswertung der inhaltlichen Aspekte. Dieser Teil besteht aus acht Fragen, anhand derer das Motivationsschreiben analysiert werden soll. Die Fragen sind so konzipiert, dass die Möglichkeit besteht, sie durch die Motivationsschreiben zu beantworten. Da aber keine Befragung durchgeführt wurde, sondern die Daten ausschließlich auf den Motivationsschreiben beruhen, bestand die Möglichkeit, dass die Fragen in dem Motivationsschreiben nicht beantwortet wurden.

Die erste Frage bezieht sich direkt auf die Motive, die zu dem Biologiestudium geführt haben. Dabei sind mögliche Motive gelistet, die zum Teil in anderen Studien nachgewiesen wurden (Ramm 2008; Unger et al. 2012). Als Zweites wird nach Arbeitserfahrungen im biologischen Bereich gefragt, gefolgt von einer Frage nach der Berufsvorstellung. Die vierte Frage bezieht sich auf die Tendenz zu einem der fünf Masterstudiengänge der Universität Innsbruck (Zoologie, Molekulare Zell- und Entwicklungsbiologie, Ökologie und Biodiversität, Mikrobiologie,

Barbara Hinger (Hg.), Zweite „Tagung der Fachdidaktik“ 2015.

Sprachsensibler Sach-Fach-Unterricht - Sprachen im Sprachunterricht.

(C) 2016 innsbruck university press, ISBN 978-3-903122-51-2, DOI 10.15203/3122-51-2 
Botanik). Mit der fünften Frage sollte die Beeinflussung der Studierenden durch die Vorlesung ins Blickfeld gerückt werden. Die fünf Qualifikationsfelder, generelle, intellektuelle Qualifikationen, experimentelle und Beobachtungsqualifikationen, zwischenmenschliche und Teamworkqualifikationen, Selbstmanagement und professionelle Entwicklungsqualifikationen' wurden in derselben Vorlesungseinheit wie die Aufgabenstellung des Motivationsschreibens behandelt. Es wurde in diesem Zusammenhang darauf hingewiesen, dass in einem Motivationsschreiben eigene Qualifikationen genannt werden sollten. Diese Frage zeigt, wie viele Studierende sich von den Ausführungen haben beeinflussen lassen und eine der Qualifikationen in ihrem Schreiben nennen. Die siebte Frage betrifft die Haltung der Studierenden. Dabei wird analysiert, ob die Studierenden in ihrem Motivationsschreiben selbstbewusst, eher arrogant oder unsicher erscheinen. Als Letztes wird noch nach dem Grund für die Wahl der Universität Innsbruck gefragt. Die statistische Auswertung erfolgte mit Microsoft ${ }^{\circledR}$ Excel ${ }^{\circledR}$ for Mac 2011. Es wurden verschiedene qualitative Hypothesen aufgestellt, die mit Hilfe des Motivationsschreibens geprüft wurden. Von den Hypothesen sollen im Rahmen des vorliegenden Beitrags drei näher betrachtet werden, die sich auf Motivation und Sprachsensitivität beziehen.

\subsection{Die Hypothesen}

\subsubsection{Motivation zur Studienwahl Biologie}

Hypothese 1a: Die Motivation der Studierenden für das Biologiestudium wird gleichermaßen von schulischen, familiären und persönlichen Parametern beeinflusst.

Hypothese 1b: Erwartungen an das Biologiestudium spielen dagegen eine geringe Rolle, ebenso wie Berufsmessen, Vorbilder, der Einfluss der Medien oder Sonstiges.

Hypothese 2: Bei den Gründen der Motivation für das Biologiestudium gibt es zwischen weiblichen und männlichen Studierenden keine signifikanten Unterschiede.

Barbara Hinger (Hg.), Zweite „Tagung der Fachdidaktik“ 2015.

Sprachsensibler Sach-Fach-Unterricht - Sprachen im Sprachunterricht.

(C) 2016 innsbruck university press, ISBN 978-3-903122-51-2, DOI 10.15203/3122-51-2 
2.3.2 Sprachkompetenz in den Motivationsschreiben

Hypothese 3: Die Textbeurteilung korreliert mit der Haltung und der Berufsvorstellung der Studierenden. Texte von Studierenden mit einer genauen Berufsvorstellung und einem selbstbewussten Auftreten in dem Motivationsschreiben werden besser beurteiltet.

\section{Ergebnisse}

\subsection{Motivation zur Studienwahl Biologie}

Bei der Frage „Was motiviert den Studierenden für das Studium der Biologie“ war es möglich, in einem Motivationsschreiben mehrere Gründe herauszufiltern. Es werden insgesamt in den 276 abgegebenen Motivationsschreiben 1247 Gründe von den im Fragebogen aufgelisteten Motiven für ein Biologiestudium

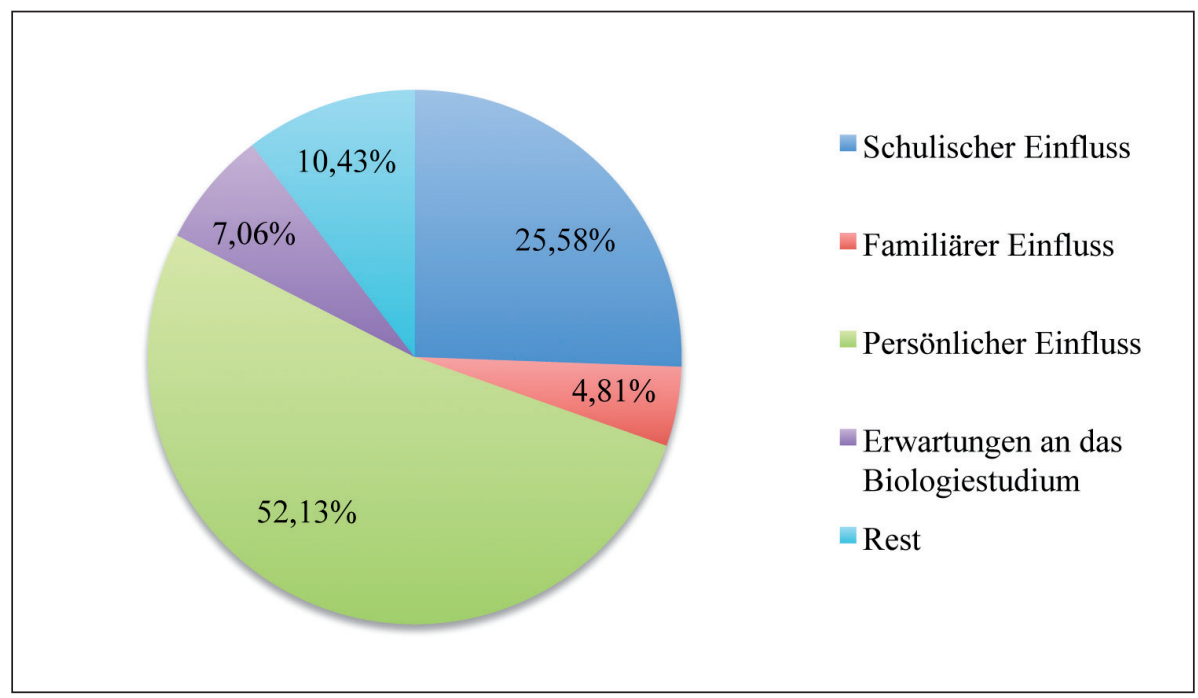

Abb. 1: Die verschiedenen Einflüsse auf Erstsemestrige des Bachelorstudiums Biologie im Wintersemester 2013/14 bei der Entscheidung für ein Biologiestudium an der Universität Innsbruck 
aufgeführt. Die verschiedenen Gründe wurden in fünf Kategorien gebündelt, wie dem schulischen und familiären Einfluss. Über die Hälfte davon wurde im Bereich des „Persönlichen Einflusses“ zusammengefasst. Mit 25,58\% ist der Einfluss durch die Schule am zweitstärksten. Dagegen scheint der Einfluss der Familie mit knapp 5\% nur sehr gering (s. Abb. 1). Dies zeigt, dass Hypothese 1 verworfen werden muss, da die Motivation der Studierenden für das Biologiestudium nicht gleichermaßen von schulischen, familiären und persönlichen Parametern beeinflusst wird, sondern der persönliche Einfluss dominiert.

In Bezug auf die gebündelten Motivationsgründe für die Entscheidung zum Biologiestudium gibt es zwischen den weiblichen und männlichen Studierenden nur einen geringen Unterschied, auch wenn die Studentinnen quantitativ betrachtet 808, die Studenten nur 439 Motive auflisteten. Für den Vergleich werden die jeweiligen Prozentsätze für die verschiedenen Kategorien errechnet. Abb. 2 zeigt die Einflüsse in den Großgruppen zusammengefasst, im Anhang werden zusätzlich in Abb. 6 die einzelnen Einflüsse aufgelistet. Zum Beispiel liegt der „Per-

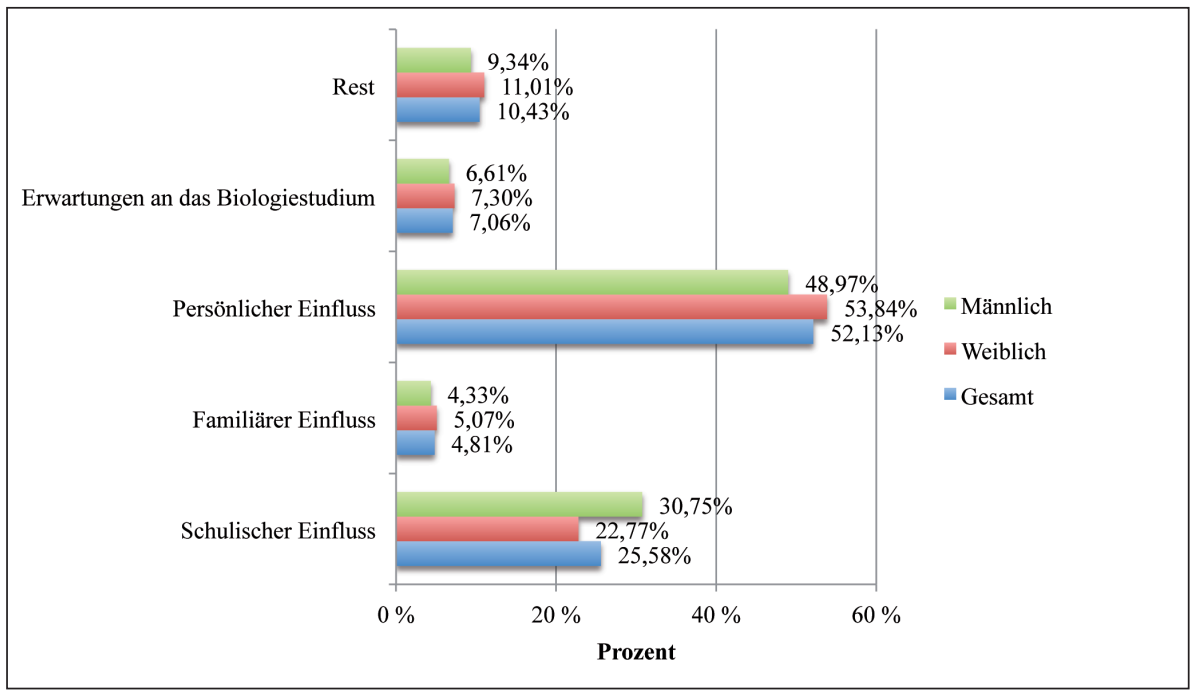

Abb. 2: Motivationsgründe der Erstsemestrigen für das Bachelorstudiums Biologie im Wintersemester 2013/14 der Universität Innsbruck. Insgesamt wurden 1247 Gründe angegeben, davon 808 von weiblichen und 439 von männlichen Studierenden. 
sönliche Einfluss" bei den männlichen Studierenden mit 48,97\% knapp unter der Hälfte, dagegen bei den weiblichen Studierenden mit 53,84\% etwas über der Hälfte. Dafür ist der „Schulische Einfluss“ bei den Studenten mit 30,75\% höher als bei den Studentinnen mit 22,77\% (s. Abb. 2). Es wurde auch ein Chi-Quadrat Test mit dem Ergebnis 0,0466 für $\mathrm{x}^{2}$ durchgeführt. Bei einem signifikanten Level von 9,488 wurde die Hypothese 2, dass es keinen signifikanten Unterschied hinsichtlich der Motivation von Studenten im Vergleich zu Studentinnen gibt, bestätigt.

\subsection{Sprachkompetenz in den Motivationsschreiben}

In Abb. 3 wird der Zusammenhang zwischen der Textbeurteilung und der Berufsvorstellung von den Studierenden gezeigt. Der Anteil der Studierenden, die eine mittelmäßige Textbeurteilung erreichen, ist bei den Studierenden mit einer genauen Berufsvorstellung mit 15,38\% relativ gering und bei den Studierenden ohne Berufsvorstellung mit 34,31\% deutlich höher.

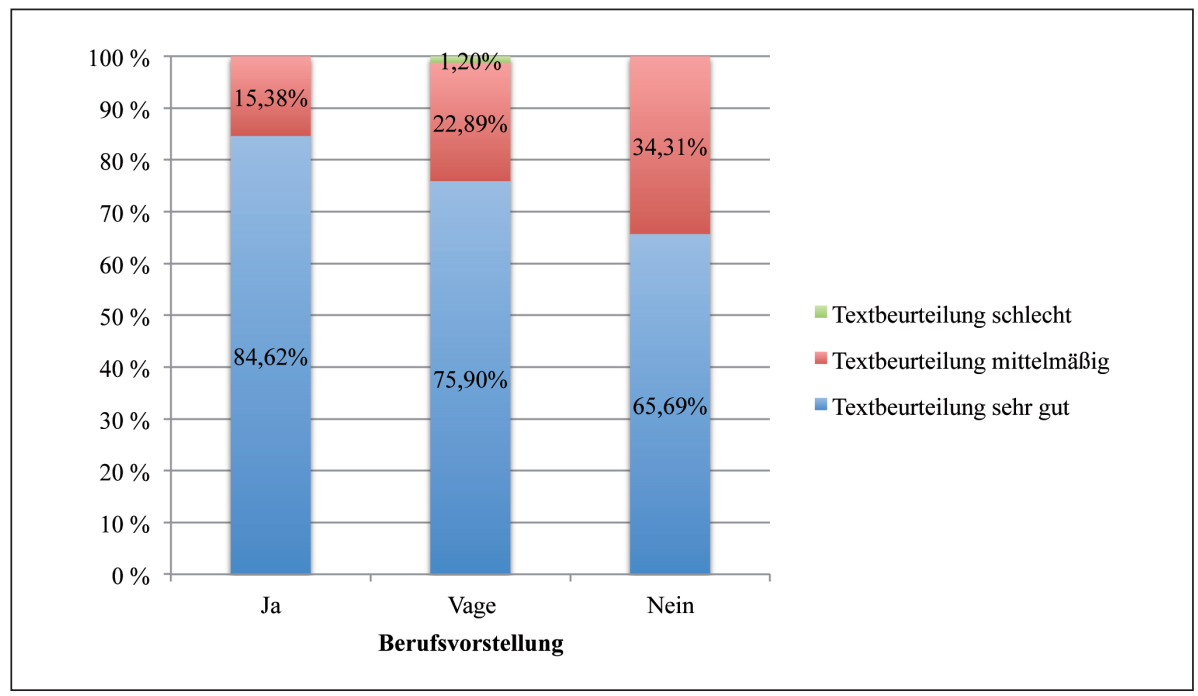

Abb. 3: Zusammenhang zwischen der Textbeurteilung und der Berufsvorstellung der Erstsemestrigen des Bachelorstudiums Biologie im Wintersemester 2013/14 der Universität Innsbruck 
Bei der Berufsvorstellung wurde zwischen drei Kategorien unterschieden: Studierende, die eine explizite Berufsvorstellung angaben, wurden der Kategorie „ja“ bei der Berufsvorstellung zugeordnet. Studierende, die zwar wussten in welche Richtung ihr späterer Beruf gehen soll, aber keinen expliziten Beruf angaben, wurden der Kategorie „vage“ zugeordnet. Die letzte Kategorie „nein“ bei der Berufsvorstellung betraf Studierende, die gar keine Berufsvorstellung angaben.

Wird der Zusammenhang zwischen der Textbeurteilung und der Haltung in den Motivationsschreiben (arrogant, selbstbewusst, unsicher) betrachtet, ist der Anteil der Studierenden mit einer mittelmäßigen Textbeurteilung bei einer arroganten $(66,67 \%)$ oder unsicheren $(72,73 \%)$ Haltung höher. Dagegen erzielten von den selbstbewussten Studierenden nur 20,55\% eine mittelmäßige Textbeurteilung (s. Abb. 4).

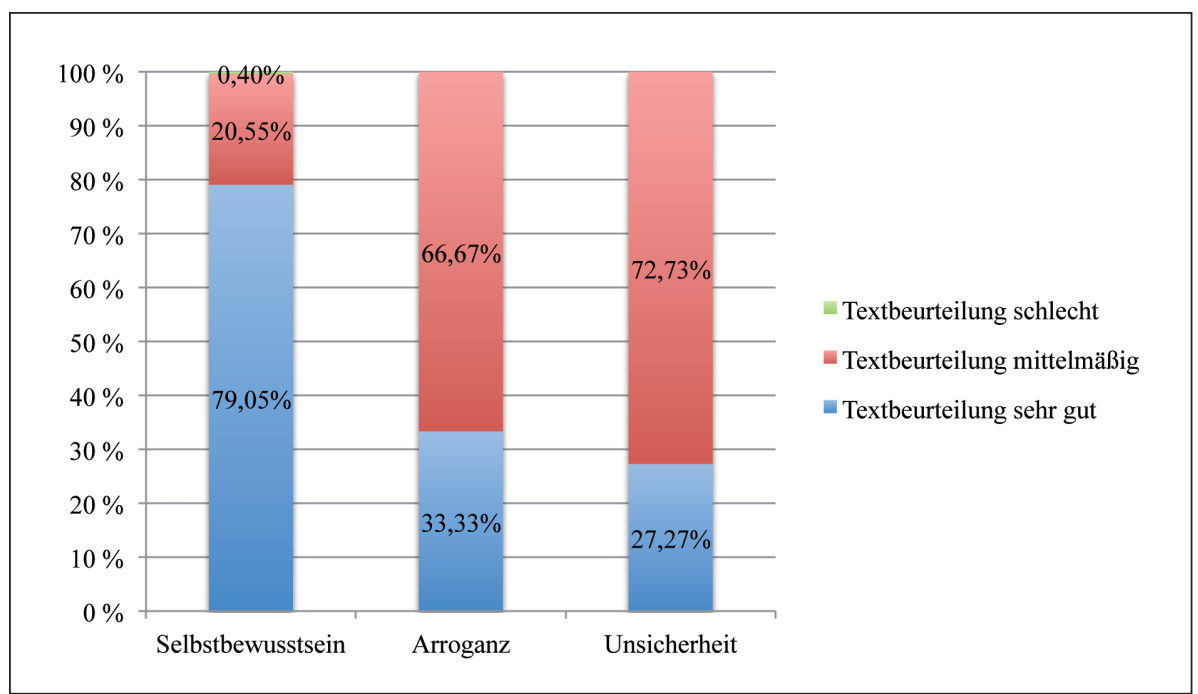

Abb. 4: Zusammenhang zwischen der Textbeurteilung und der Haltung der Erstsemestrigen des Bachelorstudiums Biologie im Wintersemester 2013/14 der Universität Innsbruck 


\section{Diskussion}

\subsection{Methodenkritik}

Mit einem Motivationsschreiben die Beweggründe und Erfolgsaussichten der Studierenden für ein Studium zu untersuchen, ist eine eher atypische Vorgehensweise. Meist werden für ähnliche Untersuchungen Fragebögen oder Befragungen durchgeführt (Bacher et al. 1994; Ramm 2008; Unger et al. 2012) oder Motivationsschreiben (motivational narrative) in Kombination mit Fragebögen herangezogen (Guzak 2012). Wird ein Motivationsschreiben zunächst ohne vordefinierten Fragebogen eingefordert, so bringt dies den Vorteil mit sich, dass die Studierenden nicht durch Fragen beeinflusst werden und nur jene Motive nennen, die für sie selbst tatsächlich relevant sind. Bei einem Fragebogen würden möglicherweise Motive suggeriert, die dem/der Studierenden gar nicht in den Sinn gekommen wären und daher möglicherweise nicht relevant für dessen/deren Motivation sind. Allerdings werden in einem Motivationsschreiben eventuell Aspekte nicht genannt, die eigentlich für eine adäquate Interpretation wichtig sind. So spielen Gründe wie die soziale Schicht und das Umfeld eine Rolle bei der Entscheidung für ein Studium (Kuh et al. 2007), was den Studierenden oft gar nicht bewusst ist. Viele Studierende haben zum Beispiel keine Begründung für die Wahl der Universität Innsbruck in den Motivationsschreiben angegeben. Für jeden Studierenden muss es aber implizit eine Begründung geben, da es sich bei der Wahl eines Studiums um eine aktive Entscheidung handelt. In der vorliegenden Untersuchung kommt eventuell zum Tragen, dass bei einem Motivationsschreiben auch darauf geachtet wird, was gut bei den Bewertenden des Motivationsschreibens ankommen könnte. So würden Studierende, wenn sie wegen ihres Freundes oder der Eltern die Universität gewählt haben, diese Begründung nicht unbedingt nennen, da sie vielleicht befürchten, dass dies einen negativen Eindruck erwecken würde. Darüber hinaus darf nicht außer Acht gelassen werden, dass die Studierenden bei einem Motivationsschreiben versuchen, sich so positiv wie möglich darzustellen und somit bei einer Befragung ein anderes Resultat erzielt werden könnte. Aus persönlichen mündlichen und schriftlichen Rückmeldungen von Studierenden an die Lehrveranstaltungsleitung wird das Verfassen eines Mo-

Barbara Hinger (Hg.), Zweite „Tagung der Fachdidaktik“ 2015.

Sprachsensibler Sach-Fach-Unterricht - Sprachen im Sprachunterricht.

(C) 2016 innsbruck university press, ISBN 978-3-903122-51-2, DOI 10.15203/3122-51-2 
tivationsschreibens als geeignet beschrieben, die Studierenden zum Nachdenken über ihre Motivation anzuregen und bei der Definition von Zielen im Studium hilfreich zu sein. Für die Auswertenden eignet es sich, die Selbstdarstellung der Studierenden zu analysieren. Um genauere Daten zu erlangen, die auch über die Motivation hinausgehen (Berufswahl, Wahl des Studienorts etc.), müssten entweder den Studierenden genauere Angaben vorgelegt werden, was das Motivationsschreiben enthalten soll, oder es müsste zusätzlich ein Fragebogen erstellt werden, der z.B. weitere Daten von den Studierenden erhebt, nachdem sie ein Motivationsschreiben verfasst haben.

\subsection{Motivation für die Studienwahl Biologie}

Hypothese 1 „Die Motivation der Studierenden für das Biologiestudium wird gleichermaßen von schulischen, familiären und persönlichen Parametern beeinflusst. Dagegen spielen Erwartungen an das Biologiestudium eine geringe Rolle, ebenso wie Berufsmessen, Vorbilder, der Einfluss der Medien oder Sonstiges", muss zum großen Teil verworfen werden. Bestätigt wurde Hypothese 1b, dass die Erwartungen an das Biologiestudium, sowie Berufsmessen, Vorbilder oder Einflüsse durch Medien kaum eine Rolle bei der Entscheidung für ein Biologiestudium zu spielen scheinen. Nicht nachgewiesen werden konnte hingegen Hypothese 1a, dass die schulischen, familiären und persönlichen Einflüsse alle gleichermaßen auf die Entscheidung einwirken. Es zeigen sich zwischen diesen Einflüssen deutliche Unterschiede, auf die im Folgenden eingegangen wird.

Bei den 250.000 Studierenden, die an den 279 Universitäten in Deutschland im Wintersemester 2006/07 in einem naturwissenschaftlichen Studium inskribiert waren, war das Fachinteresse das Hauptmotiv für ihre Studienwahl (Ramm 2008). Ebenfalls war bei der Studierenden-Sozialerhebung des Bundesministeriums für Wissenschaft und Forschung, die in Österreich im Jahr 2011 durchgeführt wurde, das wichtigste Motiv bei den österreichischen StudienanfängerInnen das Interesse am Fach (Unger et al. 2012). Die Motivation der Innsbrucker Studierenden spiegelt zum Teil diese generellen Trends bei der Entscheidung für ein Studium wider. Die persönlichen Gründe scheinen am Wichtigsten bei der Entscheidung für das Biologiestudium zu sein (s. Abb. 1) Von allen persönlichen

Barbara Hinger (Hg.), Zweite „Tagung der Fachdidaktik“ 2015.

Sprachsensibler Sach-Fach-Unterricht - Sprachen im Sprachunterricht.

(C) 2016 innsbruck university press, ISBN 978-3-903122-51-2, DOI 10.15203/3122-51-2 
Gründen sticht besonders das spezielle Fachinteresse mit 21,81\% hervor (s. Abb. 6 im Anhang).

Die schulischen Einflüsse liegen mit 25,58\% zwischen den persönlichen und familiären Einflüssen (s. Abb. 1). Diese schulischen Einflüsse dürfen nicht unterschätzt werden, da SchülerInnen, die eine positive Erfahrung mit den Naturwissenschaften in der Schule gemacht haben, eher zu einem solchen Studium tendieren als SchülerInnen, die schlechte Erfahrungen gemacht haben (Simpson \& Troost 1982).

Die familiären Einflüsse bei den Erstsemestrigen der Universität Innsbruck ist mit 4,81\% gering (s. Abb. 1). Dies widerspricht der Tatsache, dass die Eltern und die soziale Umgebung, in der ein/eine Jugendliche/r aufwächst, diese/n stark prägen und in seinen Entscheidungen beeinflussen (Simpson \& Troost 1982). Die Ursache für den geringen Prozentsatz an familiären Einflüssen in dieser Studie liegt vermutlich in der Ausrichtung der Studie. Da diese auf den Motivationsschreiben der Studierenden basiert und keine Interviews durchgeführt wurden, wurden auch keine Daten zu den Hintergründen der Studierenden, wie zur ethnischen Zugehörigkeit, zum sozialen Umfeld oder zu ihren Eltern. Bei der Wahl des Studiums spielen solche Aspekte aber durchaus eine Rolle (Kleinbeck 1975; Simpson \& Troost 1982; Unger et al. 2012). Neben dem möglichen Einfluss des Formats auf die Datenerhebung könnten die Studierenden aber auch den Einfluss ihres Elternhauses nicht bewusst als Entscheidungsfaktor bei ihrer Studienwahl wahrnehmen und deshalb nicht anführen

Bei den Innsbrucker Studierenden werden die Erwartungen an das Biologiestudium, wie die Qualität am Arbeitsplatz oder gute Aussichten auf einen sicheren Arbeitsplatz, in den Motivationsschreiben kaum als Gründe für eine Entscheidung zum Biologiestudium genannt. Bei den österreichischen StudienanfängerInnen 2011 dagegen waren nach dem Fachinteresse bessere Chancen am Arbeitsmarkt und gute Einkommensmöglichkeiten nach dem Abschluss die zwei wichtigsten Motive (Unger et al. 2012). Werden die Motive mit den deutschen Studierenden der Naturwissenschaften verglichen, ist die Motivation wieder ähnlicher wie jene der vorliegenden Studie. Dort rangieren gute Aussichten auf einen sicheren Arbeitsplatz und die Einkommenschancen auf den Plätzen vier und fünf und scheinen nur mittelmäßig wichtig zu sein. Von größerer Bedeutung

Barbara Hinger (Hg.), Zweite „Tagung der Fachdidaktik“ 2015.

Sprachsensibler Sach-Fach-Unterricht - Sprachen im Sprachunterricht.

(C) 2016 innsbruck university press, ISBN 978-3-903122-51-2, DOI 10.15203/3122-51-2 
sind die eigenen Begabungen und Fähigkeiten sowie die Vielfalt der beruflichen Möglichkeiten (Ramm 2008). Bei den Erwartungen an das Biologiestudium stechen bei den Innsbrucker Studierenden die Motive Spaß am Beruf und Vielfalt der beruflichen Möglichkeiten hervor. Dass kaum Erwartungen an das Biologiestudium als Entscheidungsfaktor genannt werden, könnte mit dem Format des Motivationsschreibens zusammenhängen. Studierende könnten solche Gründe aufgrund des Bedenkens, dass diese in einem Motivationsschreiben nicht positiv von den AdressatInnen aufgenommen werden, außer Acht lassen. Ihnen erscheinen evtl. persönliche und ideologische Begründungen für ihre Entscheidung, wie das Fachinteresse oder die Vision, die Welt zu verbessern, zweckmäßiger. Allerdings könnte dies auch mit dem Studium Biologie und den späteren Aussichten auf dem Arbeitsmarkt zusammenhängen. Der Arbeitsmarkt für BiologInnen ist schwierig, da für die Anzahl der AbsolventInnen zu wenige Stellen vorhanden sind (Ramm 2008). Wenn dies den Studierenden im Vorhinein schon bekannt ist und sie sich für das Biologiestudium entscheiden, würde das erklären, dass Motive, wie ein sicherer Arbeitsplatz, nicht ins Gewicht fallen. Interessant hierbei ist, dass bei deutschen Biologie-Studierenden die erwarteten Berufsaussichten tatsächlich eher negativ ausfallen und ein Großteil mit Schwierigkeiten bei der Stellensuche rechnet. Dagegen sind bei den anderen Naturwissenschaften die Aussichten der Studierenden eher positiv (Ramm 2008).

Wird die Motivation von Frauen und Männern verglichen, zeigt sich kein signifikanter Unterschied. Bei den weiblichen Studierenden scheint der persönliche Einfluss etwas wichtiger zu sein, dafür ist der schulische Einfluss bei den männlichen Studierenden etwas größer (s. Abb. 2). Dies bestätigt Hypothese 2, dass es bei der Motivation zwischen den Geschlechtern keinen signifikanten Unterschied gibt und spiegelt das Ergebnis der Studierenden-Sozialerhebung von 2011 wieder, bei der ebenfalls keine wesentlichen geschlechtsspezifischen Unterschiede in der Motivation festgestellt wurden (Unger et al. 2012).

\subsection{Sprachkompetenz in den Motivationsschreiben}

Bei der Textbeurteilung erzielten die Studierenden überwiegend sehr gute Beurteilungen. Der Anteil der Studierenden, die eine mittelmäßige Textbeurteilung

Barbara Hinger (Hg.), Zweite „Tagung der Fachdidaktik“ 2015.

Sprachsensibler Sach-Fach-Unterricht - Sprachen im Sprachunterricht.

(C) 2016 innsbruck university press, ISBN 978-3-903122-51-2, DOI 10.15203/3122-51-2 
erreichten, ist bei den Studierenden mit keiner genauen Berufsvorstellung doppelt so hoch wie bei den Studierenden mit einer klaren Berufsvorstellung. Bei den Studierenden die eine vage Berufsvorstellung haben, ist dieser Anteil ebenfalls größer verglichen mit jenen, die eine genaue Berufsvorstellung haben (s. Abb. 3). Dies könnte darauf verweisen, dass sich Studierende, die ein konkretes fachbezogenes Ziel vor Augen haben, mit der Formulierung ihres Motivationsschreibens leichter taten oder ihre fachbezogene Sprachkompetenz sie bei der Formulierung ihrer Ziele unterstützte. An vielen deutschsprachigen Universitäten oder Fachhochschulen werden Studienplätze nach Beurteilung eines Motivationsschreibens an BewerberInnen vergeben (Petersen \& Mery 2010). Der Trend, dem Lebenslauf eine so genannte „dritte Seite“ beizulegen, geht im deutschsprachigen Raum auf das Hesse/Schrader Bewerbungshandbuch (Hesse \& Schrader 2002) zurück, das auf ähnliche, in den USA übliche Vorgänge Bezug nimmt. Es ist anzunehmen, dass BewerberInnen mit naturwissenschaftlicher Sprachkompetenz bei der Auswahl geeigneter KandidatInnen Vorteile haben gegenüber BewerberInnen mit einer geringen fachbezogenen Sprachkompetenz. Ob die Studierenden mit guter Sprachkompetenz jedoch auch einen größeren Studienerfolg zeigen, kann im Rahmen der vorliegenden Studie nicht gezeigt werden.

Wird die Haltung der Studierenden zu der Textbeurteilung hinzugezogen, ist der Unterschied zwischen den selbstbewussten und unsicheren Studierenden groß. Von den Studierenden, die selbstbewusst erscheinen, haben nur 20,55\% eine mittelmäßige Textbeurteilung. Bei den Studierenden, die unsicher wirken, sind es dagegen $72,73 \%$, und auch bei jenen, die einen arroganten Eindruck erwecken, sind es $66,67 \%$, die nur eine mittelmäßige Beurteilung erhalten haben (s. Abb. 4). Die Unsicherheit der Studierenden spiegelt sich in ihren Motivationsschreiben wider. Glaubt man den Ausführungen von Bewerbungsratgebern, so ist ein arrogantes Auftreten in Hinsicht auf den Bewerbungserfolg nicht zielführend. Ob der hier gezeigte Zusammenhang mit gleichzeitigen Mängeln in der Sprachkompetenz eine Gefahr für den Erfolg im Studium darstellt, bedarf der Überprüfung durch Folgestudien.

Im aktuellen Kontext des Wandels der Reifeprüfung an österreichischen Schulen erhebt sich für die Autoren der vorliegenden Studie darüber hinaus die Frage, ob die Einführung der vorwissenschaftlichen Arbeit als erste Säule der neuen

Barbara Hinger (Hg.), Zweite „Tagung der Fachdidaktik“ 2015.

Sprachsensibler Sach-Fach-Unterricht - Sprachen im Sprachunterricht.

(C) 2016 innsbruck university press, ISBN 978-3-903122-51-2, DOI 10.15203/3122-51-2 
Reifeprüfung zu Veränderungen in der Bewertung der Sprachkompetenz führen und inwiefern diese eine gute Vorbereitung für ein erfolgreiches naturwissenschaftliches Studium sein kann. Dies miteinbeziehend, schlagen die Autoren ein Modell von Abhängigkeiten (s. Abb. 5) vor, das fachbezogene Sprachkompetenz als möglichen Prädiktor in einer zentralen Position zwischen Schulausbildung und Studienerfolg verortet.

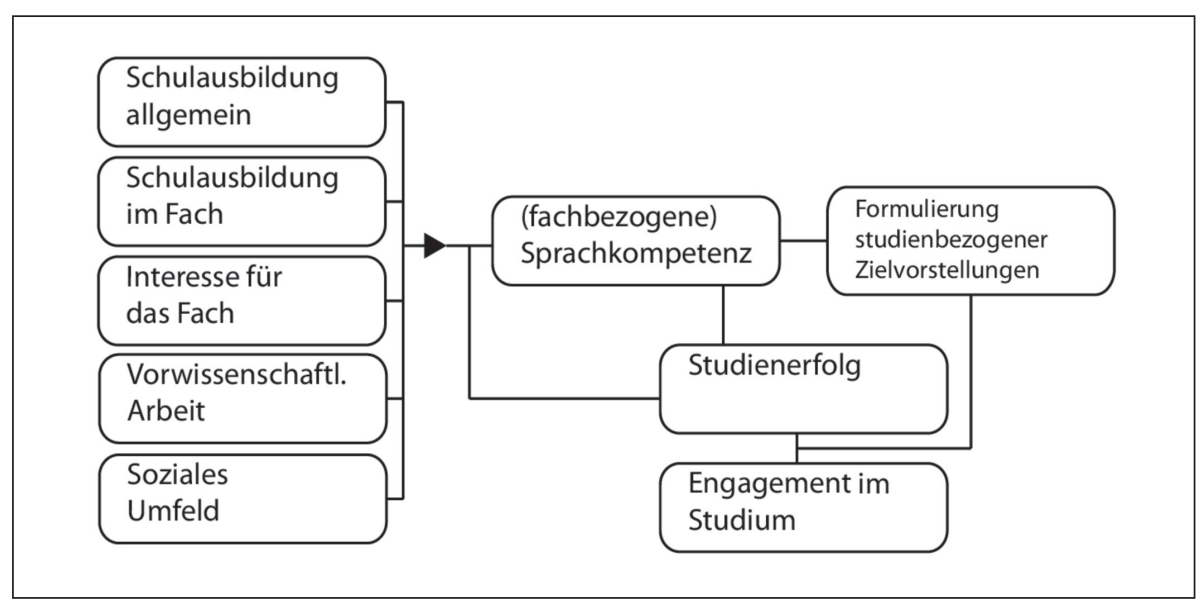

Abb. 5: Modell der Zusammenhänge zwischen Schulausbildung, sozialem Umfeld und der erlangten (fachbezogenen) Sprachkompetenz als hypothetischer Prädiktor für Erfolg im Studium. Die Verbindungslinien ohne Pfeilkopf zeigen gegenseitige Abhängigkeiten.

\section{Schlussbemerkung und Ausblick}

Die Studie zeigt, dass persönliche Gründe die Hauptmotivation für die Wahl eines Studiums sind und keine signifikanten Genderunterschiede in den Motiven nachgewiesen werden konnten. Des Weiteren zeigt sich ein Zusammenhang zwischen der Berufsvorstellung und der Sprachkompetenz. Studierende mit einer höheren Sprachkompetenz haben genauere Berufsvorstellungen. Zwischen der vermittelten Haltung der Studierenden in den Motivationsschreiben und der 
Sprachkompetenz konnte eine Relation festgestellt werden: StudentInnen mit einer selbstbewussten Haltung verfügten auch über eine bessere Sprachkompetenz.

Zukünftige Studien könnten den prädiktiven Charakter der Auswertung von Motivationsschreiben zur Studienerfolgsprognose überprüfen. Dazu könnte getestet werden, ob ein Zusammenhang zwischen Sprachkompetenz und zwischenzeitlich deaktiviertem Studium (=kein Studienerfolg) von TeilnehmerInnen der Pilotstudie besteht. Sollte sich die alleinige Auswertung der Motivationsschreiben als ungeeignet herausstellen, kann in Anlehnung an bestehende Studien (z.B. Guzak 2012) ein zusätzlicher Fragebogen erstellt werden.

\section{Literatur}

Bacher, M., Blumeberger, W., Grausgruber, A. \& Weilguni, R. (1994). Studium obne Matura. Motivation. Probleme. Studienverläufe. Eine sozialwissenschaftliche Untersuchung Studium obne Matura. Motivation. Probleme. Studienverläufe. Eine sozialwissenschaftliche Untersuchung. Linz: AK Oberösterreich.

Becker-Mrotzek, M. \& Böttcher, I. (2006). Schreibkompetenz, entwickeln und beurteilen. Berlin: Cornelsen.

Freyer, K., Epple, M., Brand, M., Schiebener, J. \& Sumfleth, E. (2014). Studienerfolgsprognose bei Erstsemesterstudierenden in Chemie. Zeitschrift für Didaktik der Naturwissenschaften, 20, 129-142.

Hellberg, B.-M. (2009). Entscheidungsfindung bei der Berufswabl. Prozessmodell der Emotionen und Kognitionen (2. Aufl.). Wiesbaden: Verlag für Sozialwissenschaften.

Guzak, K. L. (2012). Engineering design motivations: a qualitative and quantitative analysis of university students. Master`s Thesis, Michigan Technological University, Michigan. Verfügbar unter: http:// digitalcommons.mtu.edu/etds/242/ [29.03.2016].

Hesse, J. \& Schrader, H. C. (2002). Das Hesse/Schrader Bewerbungshandbuch. Köln: Eichborn-Verlag.

Kleinbeck, U. (1975). Motivation und Berufswabl. Göttingen: Verlag für Psychologie.

Kolland, F. (2002). Studienabbruch: Zwischen Kontinuität und Krise. Eine empirische Untersuchung an Österreichs Universitäten. Wien: Braumüller.

Kuh, G. D. et al. (2007): Piecing together the student success puzzle: Research, propositions and recommendations. In K. Ward \& L. Wolf-Wendel (Hrsg.), ASHE Higher Education Report, 23 (5). San Francisco: Jossey-Bass. 
Nussbaumer, M. \& Sieber, P. (1994). Texte analysieren mit dem Zürcher Textanalyseraster. In P. Sieber, E. R. Brütsch (Hrsg.), Sprachfähigkeiten - Besser als ibr Ruf und nötiger denn je! Ergebnisse und Folgerungen aus einem Forschungsprojekt (141-186). Salzburg: Sauerländer.

OECD (2013). Bildung auf einen Blick 2013. OECD-Indikatoren. Bielefeld: W. Bertelsmann Verlag.

Petersen, F. \& Mery, M. (2010). Die Bewerbung zum Studium: Erfolgreich bewerben für Bachelor und Master. Offenbach am Main: Ausbildungspark Verlag.

Ramm, M. (2008). Das Studium der Naturwissenschaften. Eine Fachmonographie aus studentischer Sicht. Bonn, Berlin: Bundesministerium für Bildung und Forschung.

Rheinberg, F. \& Vollmeyer, R. (2012). Motivation. Stuttgart: Kohlhammer.

Sieber, P. \& Brütsch, E. R. (Hrsg.) (1994). Sprachfähigkeiten - Besser als ibr Ruf und nötiger denn je! Ergebnisse und Folgerungen aus einem Forschungsprojekt. Salzburg: Sauerländer.

Simpson, R. D. \& Troost, K. M. (1982). Influences on Commitment to and Learning of Science among Adolescent Students. Science Education, 66, 763-781.

Unger, M., Dünser, L., Fessler, A. et al. (2012). Sozialerhebung 2011. Bericht zur sozialen Lage der Studierenden. Wien: Institut für Höhere Studien.

Unger, M., Wroblewski, A., Latcheva, R. et al. (2009). Frühe Studienabbrüche an Universitäten in Österreich. Endbericht. Studie im Auftrag des Bundesministeriums für Wissenschaft und Forschung BMWF. Wien: Institut für Höhere Studien.

Ziegele, F. (1997). Grundlagen der Analyse von Studienabbrüchen: Erfassung, Bewertung und Maßnahmen. Beiträge zur Hochschulforschung, 4, 435-454. 


\section{Anhang}

Tabelle 1:

Teil A. Textbeurteilung des Fragebogens zur Auswertung der Motivationsschreiben von den Erstsemestrigen des Bachelorstudiums Biologie im Wintersemester 2013/14 der Universität Innsbruck.

\begin{tabular}{|c|c|c|c|}
\hline \multicolumn{4}{|l|}{ A. Textbeurteilung } \\
\hline 1. Grundgrößen & & Aufbau & \\
\hline \multirow[t]{2}{*}{$\begin{array}{l}\text { Ist ein roter Faden } \\
\text { vorhanden? }\end{array}$} & $\mathrm{Ja}$ & $\begin{array}{l}\text { Textmuster: Wird ein der } \\
\text { Aufgabe angemessenes } \\
\text { Textmuster verwendet? }\end{array}$ & Total \\
\hline & Nein & & Nicht ganz \\
\hline 2. Sprachrichtigkeit & & & Gar nicht \\
\hline \multirow[t]{3}{*}{$\begin{array}{l}\text { Orthographie und } \\
\text { Grammatikalität }\end{array}$} & Richtig & $\begin{array}{l}\text { Textaufbau: Ist der Text } \\
\text { sinnvoll aufgebaut? Innere/ } \\
\text { Äußere Gliederung? }\end{array}$ & Total \\
\hline & $\begin{array}{l}\text { Auffallende } \\
\text { Fehler }\end{array}$ & & Nicht ganz \\
\hline & $\begin{array}{l}\text { Extrem viele } \\
\text { auffallende } \\
\text { Fehler }\end{array}$ & & Gar nicht \\
\hline $\begin{array}{l}\text { 3. Angemessenheit: } \\
\text { Verständlichkeit/Kohärenz }\end{array}$ & & $\begin{array}{l}\text { Thematische Entfaltung: } \\
\text { Wird das Thema in einer der } \\
\text { Fragestellung angemessenen } \\
\text { Art entfaltet? }\end{array}$ & Total \\
\hline \multirow[t]{2}{*}{ Wortwahl angemessen? } & Total & & Nicht ganz \\
\hline & Nicht ganz & & Gar nicht \\
\hline
\end{tabular}




\begin{tabular}{|c|c|c|c|}
\hline & Gar nicht & $\begin{array}{l}\text { Leseführung: Wird der Leser } \\
\text { aktiv durch den Text geführt? } \\
\text { Werden textstrukturierende } \\
\text { Mittel verwendet? }\end{array}$ & Total \\
\hline \multirow[t]{3}{*}{ Satzbau angemessen? } & Total & & Nicht ganz \\
\hline & Nicht ganz & & Gar nicht \\
\hline & Gar nicht & $\begin{array}{l}\text { 4. Inhaltliche Relevanz: } \\
\text { Besondere inhaltliche } \\
\text { Qualitäten }\end{array}$ & \\
\hline \multirow[t]{3}{*}{$\begin{array}{l}\text { Inhalt: Gesamtidee } \\
\text { erkennbar? }\end{array}$} & Total & $\begin{array}{l}\text { Inhaltliche Wegqualität: } \\
\text { Überzeugt der/die } \\
\text { StudentIn mit seinem } \\
\text { Motivationsschreiben? }\end{array}$ & Total \\
\hline & Nicht ganz & & Nicht ganz \\
\hline & Gar nicht & & Gar nicht \\
\hline \multirow[t]{3}{*}{ Länge angemessen? } & Total & $\begin{array}{l}\text { Inhaltliches Wagnis: } \\
\text { Lässt der Text ein besonderes } \\
\text { sprachliches Wagnis } \\
\text { erkennen? Ist er in besonderer } \\
\text { Weise kreativ? }\end{array}$ & Total \\
\hline & Nicht ganz & & Nicht ganz \\
\hline & Gar nicht & & Gar nicht \\
\hline
\end{tabular}


Tabelle 2:

Teil B. Textauswertung des Fragebogens zur Auswertung der Motivationsschreiben der Erstsemestrigen des Bachelorstudiums Biologie im Wintersemester 2013/14 der Universität Innsbruck.

\begin{tabular}{|c|c|c|c|}
\hline B. Textauswertung & & & \\
\hline $\begin{array}{l}\text { 1. Was motiviert den/die } \\
\text { StudentIn für das Studium der } \\
\text { Biologie? }\end{array}$ & Ja & $\begin{array}{l}\text { 2. Hat der/die StudentIn schon } \\
\text { einmal in einem biologischen Bereich } \\
\text { gearbeitet? }\end{array}$ & $\mathrm{Ja}$ \\
\hline Schulischer Einfluss: & & & Nein \\
\hline LehrerIn & & $\begin{array}{l}\text { 3. Hat der/die StudentIn eine } \\
\text { bestimmte Berufsvorstellung/Wunsch? }\end{array}$ & Ja \\
\hline Leistungskurswahl & & & Vage \\
\hline Schule allgemein & & & Nein \\
\hline Exkursionen & & $\begin{array}{l}\text { 4. Ist eine Tendenz zu einer der } \\
\text { fünf Masterstudiengänge, die an der } \\
\text { Universität Innsbruck angeboten } \\
\text { werden, zu erkennen? }\end{array}$ & Ja \\
\hline Familiärer Einfluss: & & & Nein \\
\hline $\begin{array}{l}\text { Familienmitglied beruflich in } \\
\text { einem naturwissenschaftlichen } \\
\text { Bereich angesiedelt }\end{array}$ & & a. Wenn ja, welche Tendenz? & \\
\hline $\begin{array}{l}\text { Naturwissenschaftliche } \\
\text { Unternehmungen mit Familie }\end{array}$ & & Botanik & \\
\hline Persönlicher Einfluss & & $\begin{array}{l}\text { Molekulare Zell- und } \\
\text { Entwicklungsbiologie }\end{array}$ & \\
\hline Spezielles Fachinteresse & & Mikrobiologie & \\
\hline
\end{tabular}




\begin{tabular}{|c|c|c|}
\hline Fester Berufswunsch & Ökologie und Biodiversität & \\
\hline $\begin{array}{l}\text { Studiensicherheit (Wollte } \\
\text { schon immer studieren) }\end{array}$ & Zoologie & \\
\hline Eigene Begabung, Fähigkeit & $\begin{array}{l}\text { 5. Werden, die in der Vorlesung genannten } \\
\text { Qualifikationen erwähnt? }\end{array}$ & $\mathrm{Ja}$ \\
\hline Auslandsaufenthalt & & Nein \\
\hline $\begin{array}{l}\text { Biologisches } \\
\text { Praktikum/FÖJ/FSJ }\end{array}$ & Wenn ja, welche Qualifikationen? & \\
\hline Naturverbundenheit & Generelle Qualifikationen & \\
\hline $\begin{array}{l}\text { Wunsch: Die Welt zu } \\
\text { verbessern }\end{array}$ & Intellektuelle Qualifikationen & \\
\hline $\begin{array}{l}\text { Erwartungen an das } \\
\text { Biologiestudium }\end{array}$ & $\begin{array}{l}\text { Experimentelle und } \\
\text { Beobachtungsqualifikationen }\end{array}$ & \\
\hline $\begin{array}{l}\text { Gute Aussichten auf sicheren } \\
\text { Arbeitsplatz }\end{array}$ & $\begin{array}{l}\text { Rechnen, Kommunikations- und IT- } \\
\text { Qualifikationen }\end{array}$ & \\
\hline $\begin{array}{l}\text { Vielfalt der beruflichen } \\
\text { Möglichkeiten }\end{array}$ & $\begin{array}{l}\text { Zwischenmenschliche und } \\
\text { Teamworkqualifikationen }\end{array}$ & \\
\hline $\begin{array}{l}\text { Einkommenschancen im } \\
\text { späteren Beruf }\end{array}$ & $\begin{array}{l}\text { Selbstmanagement und professionelle } \\
\text { Entwicklungsqualifikationen }\end{array}$ & \\
\hline $\begin{array}{l}\text { Gute Aussicht, später in } \\
\text { Führungsposition zu kommen }\end{array}$ & $\begin{array}{l}\text { 6. Welche Haltung vermittelt der/die } \\
\text { StudentIn? }\end{array}$ & $\begin{array}{l}\text { Selbstbe- } \\
\text { wusstsein }\end{array}$ \\
\hline Qualität am Arbeitsplatz & & Arroganz \\
\hline Spaß am Beruf & & Unsicherheit \\
\hline Zusatzqualifikation & $\begin{array}{l}\text { 7. Warum hat sich der/die StudentIn } \\
\text { für die Universität Innsbruck } \\
\text { entschieden? }\end{array}$ & \\
\hline
\end{tabular}




\begin{tabular}{|l|l|l|l|}
\hline $\begin{array}{l}\text { Parkstudium (Wartet auf } \\
\text { einen anderen Studienplatz) }\end{array}$ & Aus fachlichem Grund & \\
\hline $\begin{array}{l}\text { Einfluss durch Medien } \\
\text { (Fernsehen, Zeitung, Filme, } \\
\text { Bücher) }\end{array}$ & & Wegen der geographischen Lage & \\
\hline Berufsmessen/Info & & $\begin{array}{l}\text { Aufgrund einer Empfehlung (ehemalige } \\
\text { StudentInnen, Rankings etc.) }\end{array}$ & \\
\hline $\begin{array}{l}\text { Professionelle Vorbilder (z.B. } \\
\text { Nobelpreisträger, historische } \\
\text { Vorbilder) }\end{array}$ & & Sonstige & \\
\hline Sonstiges & & & \\
\hline
\end{tabular}




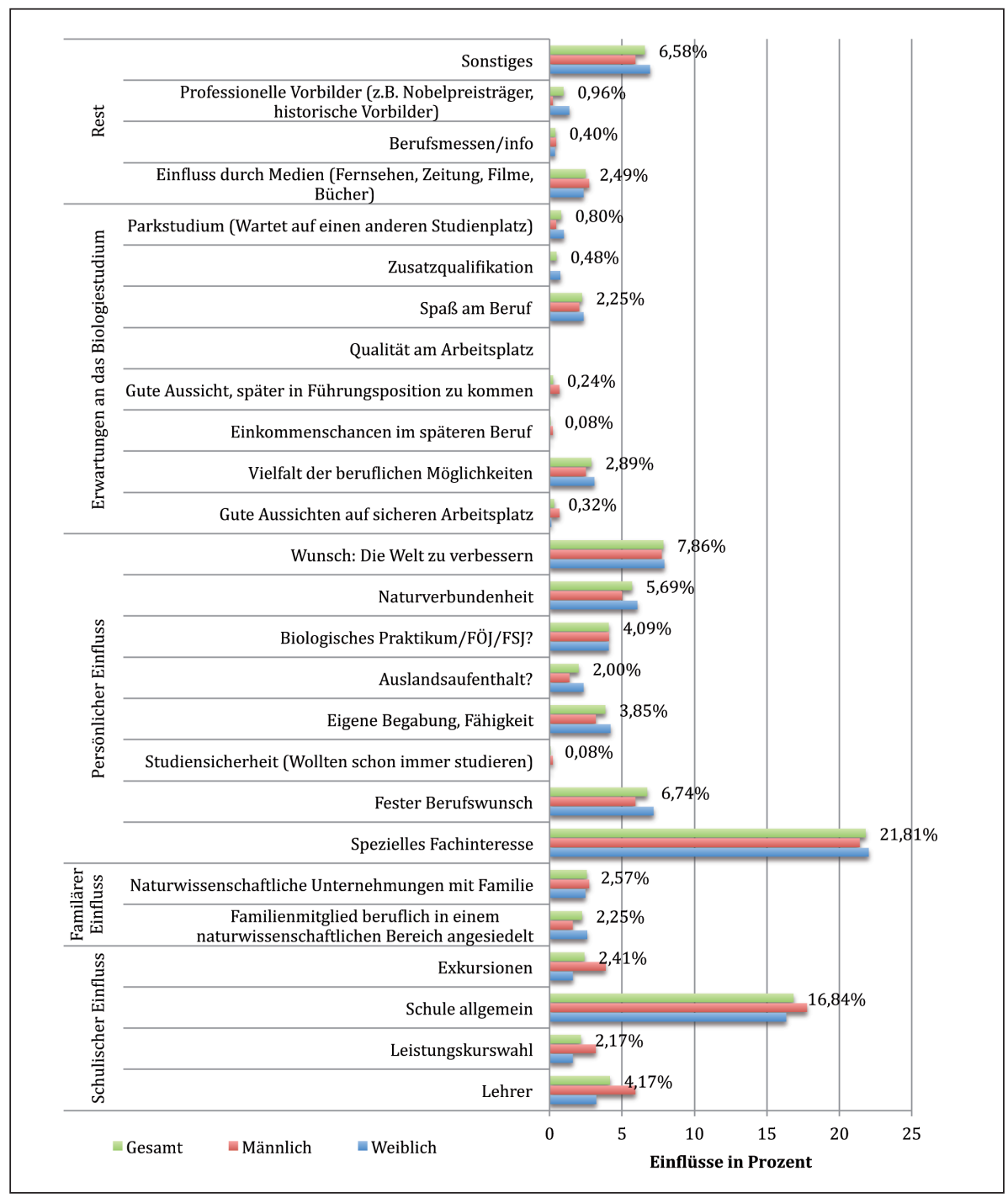

Abb. 6: Beweggründe für das Biologiestudium der Erstsemestrigen des Bachelorstudiums Biologie im Wintersemester 2013/14 der Universität Innsbruck. Die angegeben Zahlen in der Abbildung beziehen sich auf die gesamten Studierenden, die genauen Zahlen der Studentinnen und Studenten wurden nicht angegeben. 